\title{
Solid Phase Epitaxy of Ferromagnetic MnAs Layer and Quantum Dots on Annealed GaMnAs
}

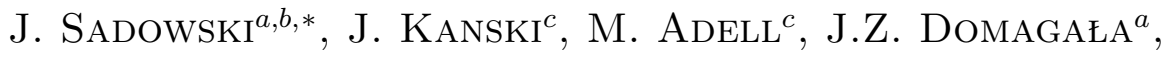

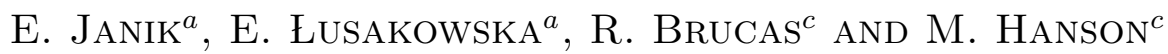 \\ ${ }^{a}$ Institute of Physics, Polish Academy of Sciences \\ al. Lotników 32/46, 02-668 Warsaw, Poland
}

${ }^{b}$ Institut für Angewandte und Experimentalle Physik, Universität Regensburg 93040 Regensburg, Germany ${ }^{c}$ Department of Applied Physics, Chalmers University of Technology 41296 Göteborg, Sweden

\begin{abstract}
We show that post growth annealing of GaMnAs under As capping at temperatures in the range of $180-210^{\circ} \mathrm{C}$ leads to significant surface modifications. Depending on GaMnAs layer thickness and composition, we obtain either a smooth continuous reacted (MnAs) surface layer or 3D islands (quantum dots). The surface modifications are due to a solid phase epitaxial process, in which Mn interstitials diffusing to the GaMnAs surface are bound with the As.
\end{abstract}

PACS numbers: 81.10.Jt, 81.15.Hi, 68.65.Hb, 68.37.Ps

\section{Introduction}

Post growth annealing (PGA) of GaMnAs is an established procedure to increase the ferromagnetic phase transition temperature $\left(T_{\mathrm{c}}\right)$. The increased $T_{\mathrm{c}}$ is ascribed to two co-operating effects, both related to removal of $\mathrm{Mn}$ atoms located at interstitial sites $\left(\mathrm{Mn}_{\mathrm{I}}\right)$. Along with other defects like As antisites $\left(\mathrm{As}_{\mathrm{Ga}}\right)$ the Mn interstitials are abundant in the as-grown GaMnAs (around $20 \%$ of the total Mn concentration), because the GaMnAs must be prepared by MBE growth at temperatures not exceeding $200-280^{\circ} \mathrm{C}$ (the higher $\mathrm{Mn}$ content, the lower growth temperature is necessary). The $\mathrm{Mn}$ interstitials counteract the stability of the

*corresponding author; e-mail: janusz.sadowski@physik.uni-regensburg.de 
ferromagnetic state by acting as double donors (thereby compensating the valence band holes responsible for ferromagnetic ordering), and by directly coupling antiferromagnetically to $\mathrm{Mn}$ in Ga substitutional sites (which provide both the magnetic moments and valence band holes). It has been reported by several groups [1-5] that by post-growth annealing the $T_{\mathrm{c}}$ of GaMnAs can be significantly increased, up to $160-170 \mathrm{~K}$, whereas in as-grown material it is usually below 100-110 K. It is generally accepted that PGA changes the defect structure of GaMnAs. So far it was proposed, and partially verified experimentally $[6,7]$ that PGA mainly affects the $\mathrm{Mn}_{\mathrm{I}}$ defects. In the case of $\mathrm{Ga}_{0.95} \mathrm{Mn}_{0.05} \mathrm{As}$ the $\mathrm{Mn}_{\mathrm{I}}$ concentration is thus reduced from about $1 \%$ down to below $0.1 \%$ after the PGA procedure. The $\mathrm{As}_{\mathrm{Ga}}$ is also a double donor, and can reach concentrations up to 0.5\%, as shown for LT GaAs (grown at the same low temperatures as GaMnAs), and also directly for GaMnAs [8, 9]. With the above-mentioned concentrations, the compensation due to the two most prevalent double donor defects can exceed $50 \%$ in $\mathrm{Ga}_{0.95} \mathrm{Mn}_{0.05}$ As.

The optimum PGA treatments are at temperatures in the range of $180-260^{\circ} \mathrm{C}$ and periods ranging from $30 \mathrm{~min}$ to $100 \mathrm{hrs}$. It is currently debated which PGA procedures lead to the best results [1-6, 10-13], however it is important to note that GaMnAs is a metastable compound and by extended annealings at temperatures exceeding $280-300^{\circ} \mathrm{C}$ decomposition occurs into MnAs inclusions inside the GaAs matrix. The PGA process shows some peculiarities, and it is not clear whether the main mechanism for removing $\mathrm{Mn}_{\mathrm{I}}$ is some volume segregation process $[7,13]$, or only out-diffusion and surface passivation. There are suggestions that both processes take place, the first one being important in case of thick GaMnAs samples (with thicknesses higher than about $0.5 \mu \mathrm{m}$ ), the other process (out-diffusion and surface passivation) was demonstrated to be dominating in thin GaMnAs (layers thinner than $0.1 \mu \mathrm{m}$ ). However, so far almost nothing is known about the changes of GaMnAs surface due the PGA processes. Here we show that, irrespectively of the GaMnAs thickness, PGA always leads to $\mathrm{Mn}_{\mathrm{I}}$ out-diffusion. Moreover, having suitably prepared GaMnAs surface, we were able to use the out-diffused Mn to form ferromagnetic quantum dots in a novel process.

\section{Samples preparation}

The GaMnAs samples were grown by the LT MBE technique at MAX-Lab, Lund University, Sweden. The details of the growth were described elsewhere [5]. After the GaMnAs growth the substrate temperature was decreased to below $100^{\circ} \mathrm{C}$, and a thick amorphous arsenic layer was deposited on the GaMnAs surface. After the As capping deposition the samples were subjected to the PGA process, either in the MBE system, directly after the capping layer deposition, or in air. We have shown previously [5] that this PGA process is much more efficient than annealing without As capping (the effects are achieved after much shorter annealing times). In the present study we have applied the PGA procedures only 
in vacuum (at $10^{-9}$ mbar) in the MBE system, with effusion cell shutters closed and effusion cells temperatures lowered to the standby values. The PGA processes were performed in two steps: first the $3 \mathrm{~h}$ annealings at temperatures of $180-220^{\circ} \mathrm{C}$ were done, under control of the surface by reflection high energy electron diffraction (RHEED). The GaMnAs surface with As capping is featureless in RHEED pictures (a diffuse constant intensity background is observed). After this first annealing stage, if the amorphous As layer was not entirely desorbed from the GaMnAs surface, the sample temperature was gradually increased in $15-20^{\circ} \mathrm{C}$ steps. This temperature increase was stopped at the appearance of monocrystalline diffraction pattern, which took place at a temperature in the range of $250-350^{\circ} \mathrm{C}$, depending on the sample. Usually this additional annealing took about 1-2 hrs, but in some samples As capping desorbed entirely already after the first annealing stage. The decapping is of course only applied to generate a well-ordered surface for a further epitaxial growth, but it should be stressed that the initial annealing is sufficient to achieve optimal magnetic properties as verified by comparison between decapped samples and samples not subjected to the additional decapping treatment.

\section{Modifications of GaMnAs surfaces upon annealing}

Figure 1 shows the surface morphologies of four $\mathrm{Ga}_{0.95} \mathrm{Mn}_{0.05} \mathrm{As}$ samples with different thicknesses (1.0, 0.3, 0.2, and $0.15 \mu \mathrm{m}$ - samples a, b, c, and d) as observed by AFM. Considering the preparation method, it is most likely that the clearly visible small dots on the decapped GaMnAs surface are formed by reaction between surface out-diffused Mn interstitials and amorphous arsenic capping, and thus composed of MnAs. The interesting feature, present in all the AFM pictures is the preferential arrangement of the MnAs dots on ring-like structures. The diameter of these structures increases with the decreasing thickness of GaMnAs layer, from which Mn interstitials were out-diffused. The average density of dots is thus lower on the thinner layers. This is simply due to the reduced amount of Mn interstitials within the GaMnAs layers. The thinnest sample (Fig. 1d) does not follow this trend, which might be connected to a slightly different procedure of the decapping step. We have verified by TEM measurements [14] that the crystalline structure of the dots seen in Fig. 1 is hexagonal, so we conclude that they are composed of MnAs in its natural, NiAs-type crystal structure. The dots in our TEM pictures resemble in fact the hexagonal MnAs inclusions found in hightemperature annealed GaMnAs [15]. MnAs inclusions [15] and surface dots [16] can also have a zinc-blende (ZB) structure imposed by GaAs, but only in the case of much smaller dimensions, $5-10$ times smaller than surface MnAs dots discussed here. This is consistent with theoretical predictions showing that MnAs is not stable in the cubic, zinc-blende phase [17].

With reduced GaMnAs thickness the amount of out-diffused $\mathrm{Mn}_{\mathrm{I}}$ decreases and below some thickness, the formation of MnAs dots is inhibited. This critical 

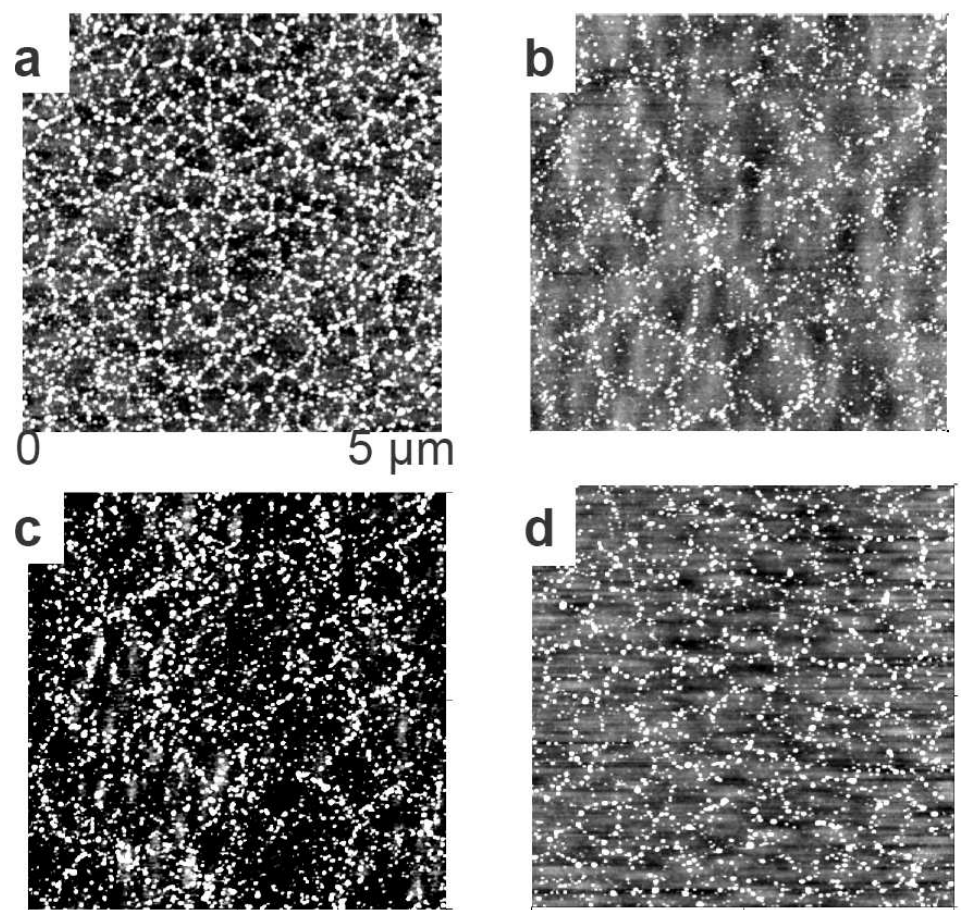

Fig. 1. Surface morphologies of $\mathrm{MnAs}$ dots on $\mathrm{Ga}_{0.95} \mathrm{Mn}_{0.05} \mathrm{As}$ layers with different thicknesses: $1 \mu \mathrm{m}$ (a), $0.3 \mu \mathrm{m}$ (b), $0.2 \mu \mathrm{m}$ (c), $0.15 \mu \mathrm{m}$ (d).

thickness depends on the Mn content in the as-grown GaMnAs layer. Without MnAs dots the annealed surface is smooth (see AFM picture in Fig. 2, taken for annealed $200 \AA$ thick $\mathrm{Ga}_{0.935} \mathrm{Mn}_{0.065} \mathrm{As}$ ) and shows RHEED diffraction streaks identical to those of the GaMnAs surface. However, we know from other measurements $[14,18]$ that this surface is very Mn rich. Thus we postulate that in this case MnAs forms a continuous, zinc-blende surface layer. This is supported by resonance photoemission spectra, which we find to be very similar to corresponding spectra from ZB MnAs dots. The thickness of this layer $\left(d_{\mathrm{MnAs}}\right)$ is unknown, but assuming that all the Mn interstitials are out-diffused and bound with As at the surface in a zinc-blende structure layer with the same lattice constant as the underlying GaAs, $d_{\mathrm{MnAs}}$ can be 1 to 2 molecular layers $(2.8$ to $5.6 \AA$ ). There is some periodical surface modulation, clearly visible in Fig. 2. This resembles coexisting $\alpha$ and $\beta$ phases present in hexagonal MnAs layers deposited on the GaAs(100) substrates [19]. In our case the RHEED images demonstrate that there is only one crystalline phase on the surface, moreover it exhibits exactly the same diffraction patterns as GaMnAs surface.

In order to examine the magnetic properties of the MnAs dots we have done room temperature magnetic force microscope (MFM) measurements of a $0.2 \mu \mathrm{m}$ thick $\mathrm{Ga}_{0.95} \mathrm{Mn}_{0.05}$ As sample, subjected to the PGA described above. AFM and 


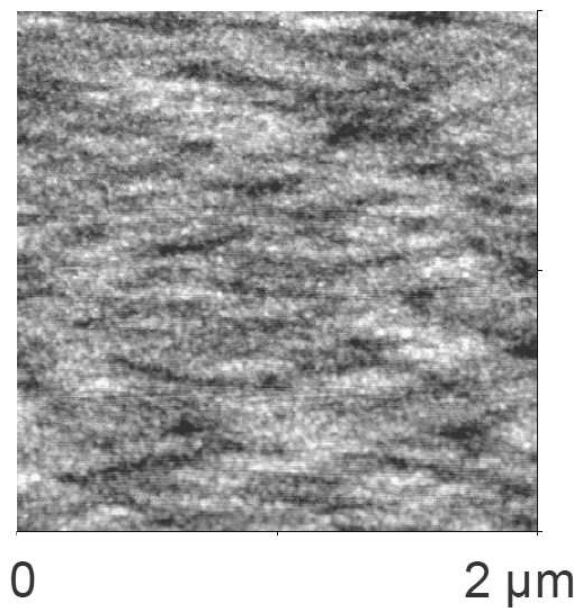

Fig. 2. AFM surface morphology of $200 \AA$ thick $\mathrm{Ga}_{0.935} \mathrm{Mn}_{0.065}$ As layer with smooth surface after annealing and amorphous arsenic capping desorption. The surface roughness is at the level of $1 \mathrm{ML}(2.8 \AA)$, some modulation of the Mn-rich surface layer is visible.
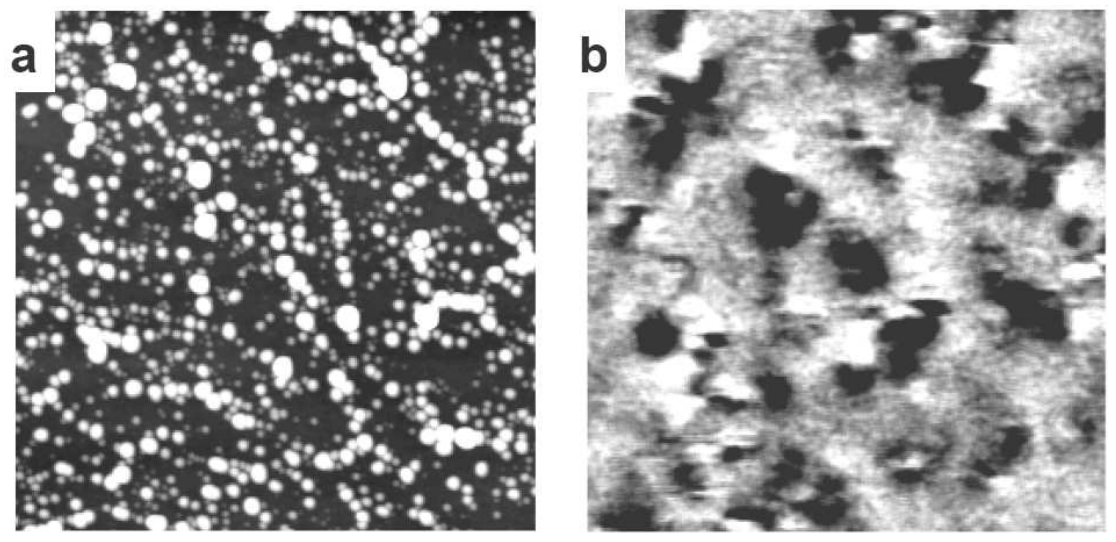

0

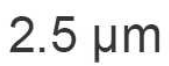

Fig. 3. (a) AFM and (b) MFM images from the same surface region of annealed $0.2 \mu \mathrm{m}$ thick $\mathrm{Ga}_{0.95} \mathrm{Mn}_{0.05}$ As layer with MnAs dots on the surface.

MFM images of the same region of the sample are shown in Fig. 3. It can be seen that the biggest dots give clear magnetic contrast appearing as dark spots. However the spatial resolution is less good in MFM than AFM, and the magnetic contrast represents ensembles of dots rather than individual objects. No magnetic contrast is seen for the smaller dots, which means that they are either non-magnetic at room temperature, or are magnetically too soft to be probed with the MFM tip. At this point we are not able to distinguish between these two situations. 


\section{Influence of annealing on the GaMnAs properties}

As stated above, the post growth annealing effectively removes $\mathrm{Mn}_{\mathrm{I}}$ defects from GaMnAs volume. This is associated with an increase in both $T_{\mathrm{c}}$ and concentration of holes, and also a significant decrease in the lattice constant $[5,9]$. Figure 4 shows the results of X-ray diffraction measurements of the sample presented in Fig. 1d, the $0.15 \mu \mathrm{m}$ thick $\mathrm{Ga}_{0.95} \mathrm{Mn}_{0.05}$ As layer. After the annealing process the angular position of GaMnAs 004 Bragg reflection is significantly shifted

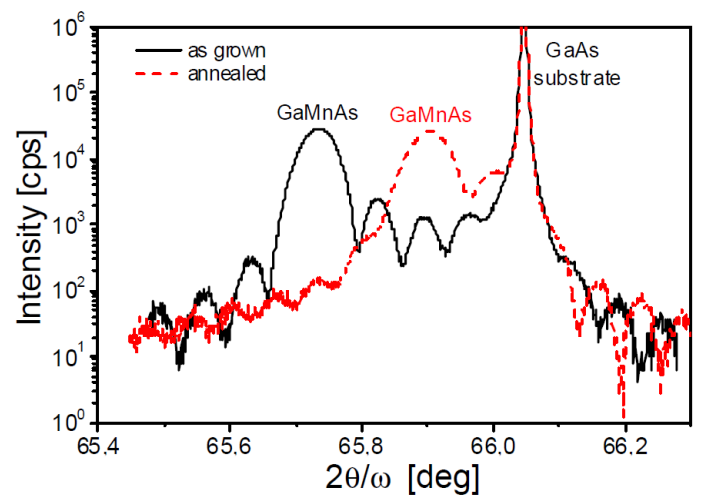

Fig. 4. X-ray (004) Bragg reflection from $0.15 \mu \mathrm{m}$ thick $\mathrm{Ga}_{0.95} \mathrm{Mn}_{0.05}$ As layer before annealing (solid curve) and after annealing at high vacuum (dashed curve) leading to the out diffusion of Mn interstitials from GaMnAs bulk and formation of MnAs dots on the surface. Diffusion of Mn interstitials reduces the GaMnAs lattice parameter, evidenced by the significant shift of (004) GaMnAs reflection towards higher diffraction angles.

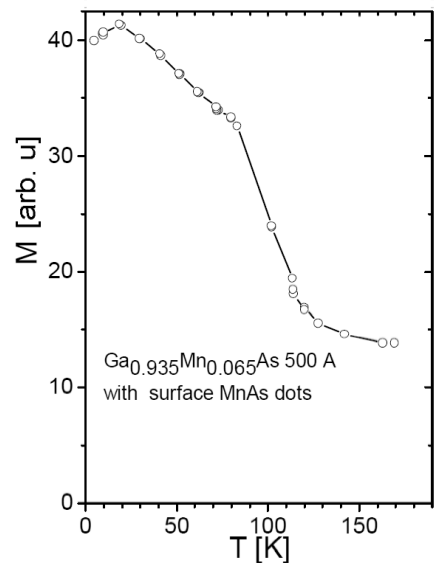

Fig. 5. Temperature dependence of magnetization for $500 \AA$ thick $\mathrm{Ga}_{0.935} \mathrm{Mn}_{0.065} \mathrm{As}$ layer after annealing and arsenic capping desorption. The high temperature magnetization background is due to the ferromagnetic phase of surface MnAs. 
towards higher diffraction angles, which means the lattice constant reduction due to the removal of $\mathrm{Mn}_{\mathrm{I}}$ defects. The effect of $\mathrm{Mn}_{\mathrm{I}}$ removal is reflected in improved magnetic properties. Figure 5 presents the temperature dependence of magnetization for a $500 \AA$ thick $\mathrm{Ga}_{0.935} \mathrm{Mn}_{0.065}$ As sample with MnAs dots, after PGA procedure and complete decapping, measured at an external magnetic field of 30 Oe applied along the [010] azimuth of the (001) sample plane. The magnetization starts to rise at close to $160 \mathrm{~K}$, due to the appearance of GaMnAs FM phase. The significant magnetization background at high temperatures can be associated with the surface MnAs magnetic phase.

\section{Conclusions}

We have shown that low temperature post-growth annealing of GaMnAs in the presence of amorphous arsenic capping leads to reaction between out-diffused Mn interstitials and surface arsenic. Depending on starting conditions, the annealed layer is terminated either by an ultrathin smooth epitaxial layer, presumably zinc-blende MnAs, or decorated with MnAs quantum dots in a ring-like pattern. The transition between the two situations is controlled by the amount of Mn interstitials in the GaMnAs layer, i.e. by the layer thickness and the Mn concentration. The ring-like organization of the MnAs dots may be related to surface strain modulation in the MnAs wetting layer.

\section{References}

[1] K.W. Edmonds, K.Y. Wang, R.P. Campion, A.C. Neumann, N.R. S. Farley, B.L. Gallagher, C.T. Foxon, Appl. Phys. Lett. 81, 4991 (2002).

[2] K.C. Ku, S.J. Potashnik, R.F. Wang, S.H. Chun, P. Schiffer, N. Samarth, M.J. Seong, A. Mascarenhas, E. Johnston-Halperin, R.C. Myers, A.C. Gossard, D.D. Awschalom, Appl. Phys. Lett. 82, 2302 (2003).

[3] D. Chiba, K. Takamura, F. Matsukura, H. Ohno, Appl. Phys. Lett. 82, 3020 (2003).

[4] M. Malfait, J. Vanacken, V.V. Moshchalkov, W. Van Roy, G. Borghs, Appl. Phys. Lett. 86, 132501 (2005).

[5] M. Adell, L. Ilver, J. Kanski, V. Stanciu, P. Svedlindh, J. Sadowski, J.Z. Domagala, F. Terki, C. Hernandez, S. Charar, Appl. Phys. Lett. 86, 112501 (2005).

[6] B.J. Kirby, J.A. Borchers, J. J. Rhyne, S.G.E. te Velthuis, A. Hoffmann, K.V. O'Donovan, T. Wojtowicz, X. Liu, W.L. Lim, J.K. Furdyna, Phys. Rev. $B$ 69, 081307 (2004).

[7] K.M. Yu, W. Walukiewicz, T. Wojtowicz, J. Denlinger, M.A. Scarpulla, X. Liu, J.K. Furdyna, Appl. Phys. Lett. 86, 042102 (2005).

[8] X. Liu, A. Prasad, J. Nishio, E.R. Weber, Z. Liliental-Weber, W. Walukiewicz, Appl. Phys. Lett. 67, 279 (1995).

[9] J. Sadowski, J.Z. Domagala, Phys. Rev. B 69, 075206 (2004). 
[10] M. Adell, J. Kanski, L. Ilver, J. Sadowski, V. Stanciu, P. Svedlindh, Phys. Rev. Lett. 94, 139701 (2005).

[11] K.W. Edmonds, P. Bogusławski, K.Y. Wang, R.P. Campion, S.V. Novikov, N.R.S. Farley, B.L. Gallagher, C.T. Foxon, M. Sawicki, T. Dietl, M. Buongiorno Nardelli, J. Bernholc, Phys. Rev. Lett. 94, 139702 (2005).

[12] K.W. Edmonds, P. Boguslawski, K.Y. Wang, R.P. Campion, S.N. Novikov, N.R.S. Farley, B.L. Gallagher, C.T. Foxon, M. Sawicki, T. Dietl, M. Buongiorno Nardelli, J. Bernholc, Phys. Rev. Lett. 92, 037201 (2004).

[13] W. Limmer, A. Koeder, S. Frank, V. Avrutin, W. Schoch, R. Sauer, K. Zuern, J. Eisenmenger, P. Ziemann, E. Peiner, A. Waag, Phys. Rev. B 71, 205213 (2005).

[14] J. Sadowski, M. Adell, J. Kanski, L. Ilver, E. Janik, E. Lusakowska, J.Z. Domagala, S. Kret, P. Dluzewski, R. Brucas, M. Hanson, submitted to Appl. Phys. Lett.

[15] M. Moreno, A. Trampert, B. Jenichen, L. Daweritz, K.H. Ploog, J. Appl. Phys. 92, 4672 (2002).

[16] J. Okabayashi, M. Mizuguchi, K. Ono, M. Oshima, A. Fujimori, H. Kuramochi, H. Akinaga, Phys. Rev. B 70, 233305 (2004).

[17] S. Sanvito, N.A. Hill, Phys. Rev. B 62, 15553 (2000).

[18] M. Adell, L. Ilver, J. Kanski, J. Sadowski, R. Mathieu, V. Stanciu, Phys. Rev. B 70, 125204 (2004).

[19] T. Plake, M. Remsteiner, V.M. Kaganer, B. Jenichen, M. Kästner, L. Däweritz, K.H. Ploog, Appl. Phys. Lett. 80, 2523 (2002). 